\title{
DISEÑO DE UNA SUMADORA ELÉCTRICA COMO PROTOTIPO DIDÁCTICO
}

\author{
Design of an electric adder as didactic prototype
}

EPISTEMUS

ISSN: 2007-8196 (electrónico)

ISSN: 2007-4530 (impresa)

Héctor Alfredo Hernández Hernández ${ }^{1}$ Jesús Francisco Espinoza Fierro ${ }^{2}$

Recibido: 30 de agosto de 2016

Aceptado: 9 de diciembre de 2016

Autor de Correspondencia:

M.C. Héctor Alfredo Hernández Hernández

Correo:hector@mat.uson.mx

\section{Resumen}

En este trabajo se presenta el diseño de un prototipo didáctico que suma un par de números enteros. La operación a destacar es la suma en el sistema binario y en su elaboración se utilizan elementos simples y de bajo costo. Se establecieron restricciones en el rango de las posibles sumas y se presentan las configuraciones que deben tener los circuitos que realizan las conversiones del sistema decimal al sistema binario, y viceversa, con el fin de que estos últimos no resulten mucho más complicados que el circuito de interés principal. La utilización de herramientas de corte de control numérico fue imprescindible para la elaboración física de este prototipo didáctico.

Palabras clave: Sumadora, prototipo, didáctico.

\section{Abstract}

This work presents the design of a didactic prototype that adds a couple of integer numbers, the operation highlight is the sum in the binary system, and to design it are needed simple and low cost elements. Some restrictions are given on the range of possible sums and also we present the configurations of circuits that perform conversions decimal to binary system and vice versa so that the latter will not be much more complicated than the circuit of main interest was found. The use of cutting tools of numerical control were essential to develop this educational prototype.

Keywords: Adder, prototype, didactic. 


\section{INTRODUCCIÓN}

Cuando se busca promover la ciencia y el conocimiento entre la sociedad, es importante elaborar actividades o construir dispositivos que resulten atractivos al público en general, sin perder de vista que existe una amplia diversidad de gustos y preferencias. Si al atender alguna exposición de ciencia se observa que al menos una pequeña fracción del público asistente se emociona, manifiesta asombro o simplemente le agrada, se considera que se ha cumplido el objetivo modestamente.

A través de la historia el ser humano ha creado una gran cantidad de instrumentos de cálculo motivado principalmente por la necesidad de llevar a cabo operaciones aritméticas con mayor velocidad y precisión. Existen dispositivos de muchos estilos, desde los mecánicos hasta los electrónicos donde se manifiesta el ingenio del ser humano. En [1] y [2, Apéndice D] puede consultarse más extensamente sobre la evolución de la calculadora y la computadora, desde sus inicios hasta como la conocemos actualmente.

En este trabajo presentamos el diseño de una sumadora eléctrica con fines didácticos. Consideramos que el instrumento diseñado tiene características destacables que lo recomiendan como un dispositivo que debiéramos replicar y exhibir en el aula o en eventos de divulgación de la ciencia.

La sumadora recibe un par de números elegidos del cero al diez y regresa como resultado la suma de dichos números. El funcionamiento de este dispositivo requiere una completa interacción con el usuario, lo cual le permite comprender detalladamente el proceso que se lleva a cabo, además los materiales utilizados para su construcción son relativamente fáciles de adquirir.

No se pretende dar una explicación muy detallada del dispositivo, la idea es que quien lea este trabajo y posea un poco de experiencia en circuitos lógicos tenga la oportunidad de construir uno propio, o al menos tomar ideas para diseñar un instrumento similar. Mostramos brevemente algunos detalles importantes suponiendo que el lector no está completamente familiarizado con el tema. Construir sumadoras no es una actividad novedosa, suele ser un proyecto para ser desarrollado por estudiantes de ingeniería, sin embargo es bastante común que utilicen dispositivos sofisticados en su elaboración. En [3] y [4], se muestra la construcción de un prototipo similar al presentado en este trabajo usando compuertas lógicas. La idea de construir circuitos lógicos generando las compuertas lógicas requeridas utilizando sólo interruptores no es nueva, pero gracias a la introducción de lo que hemos llamado relevadores manuales, ha hecho posible la elaboración de este instrumento y estamos seguros que servirá para diseñar muchos otros con las características que aquí resaltamos, entre ellas la alta credibilidad.

El lector puede adentrase un poco más en el tema y constatar que la suma forma parte importante de las otras tres operaciones básicas, a saber; la multiplicación, la resta y la división de números binarios, de ahí la importancia de centrar los esfuerzos en construir un prototipo que realice la suma de dos números.

\section{PRELIMINARES}

El sistema de numeración que utilizamos con mayor frecuencia es el sistema decimal en el que se utilizan diez dígitos $D=\{0,1,2,3,4,5,6,7,8,9\}$. También existen otros sistemas que resultan ser muy útiles, entre ellos el sistema binario, que requiere de sólo dos dígitos $B=\{0,1\}$. Ambos sistemas utilizan una notación posicional. En [5] el autor expone un desarrollo histórico muy interesante sobre el sistema binario y otros sistemas de numeración, su lectura es ampliamente recomendada.

La siguiente tabla muestra las equivalencias en el sistema binario de los primeros 21 números naturales.

Tabla 1. Equivalencias decimal-binario.

\begin{tabular}{|c|c|c|c|}
\hline Decimal & Binario & Decimal & Binario \\
\hline 0 & 0000 & 11 & 1011 \\
\hline 1 & 0001 & 12 & 1100 \\
\hline 2 & 0010 & 13 & 1101 \\
\hline 3 & 0011 & 14 & 1110 \\
\hline 4 & 0100 & 15 & 1111 \\
\hline 5 & 0101 & 16 & 10000 \\
\hline 6 & 0110 & 17 & 10001 \\
\hline 7 & 0111 & 18 & 10010 \\
\hline 8 & 1000 & 19 & 10011 \\
\hline 9 & 1001 & 20 & 10100 \\
\hline 10 & 1010 & 21 & 10101 \\
\hline
\end{tabular}

También en el sistema binario cada dígito representa un valor según la posición que ocupe en la secuencia, asíal número 13 le corresponde la secuencia en sistema binario 1101 debido a que $13=1\left(2^{3}\right)+1\left(2^{2}\right)+0\left(2^{1}\right)+1\left(2^{0}\right)$.

En [6] puede consultarse una excelente introducción al álgebra booleana, la cual puede pensarse como el álgebra de un sistema de dos elementos ( 0 y 1 ) con los operadores binarios AND y OR, así como la operación unaria de complemento NOT. El álgebra booleana tiene una aplicación natural en la teoría de circuitos de conmutación eléctrica; Whitesitt [6, Cap. 6] muestra una configuración mediante compuertas lógicas para la suma y resta de números en el sistema binario, lo cual esté estrechamente relacionado al presente trabajo.

Es deseable que el lector esté familiarizado con la suma de números en el sistema binario para que comprenda el funcionamiento del instrumento propuesto. Afortunadamente lo anterior no es un requisito para poder construir la sumadora. 


\section{Ejemplo. Suma en el sistema binario y acarreo.}

En la Figura 1 se muestra un ejemplo para la suma de dos números en el sistema binario.

\begin{tabular}{|l|ll|}
\hline $\mathrm{a}$ & 0 & 1 \\
\hline 0 & 0 & 0 \\
1 & 0 & 1 \\
\hline &
\end{tabular}$\quad$\begin{tabular}{|l|ll|}
++ & 0 & 1 \\
\hline 0 & 0 & 1 \\
1 & 1 & 0
\end{tabular}$\quad$\begin{tabular}{rlllll|}
1 \\
1
\end{tabular}$\quad$\begin{tabular}{lllllll}
1 & 1 & 0 & 0 & 1 \\
1 & 1 & 0 & 1 & 0 \\
\hline 1 & 1 & 0 & 0 & 1 & 1
\end{tabular}

Figura 1. Ejemplo para sumar dos números binarios.

El cálculo realizado a la derecha de la Figura 1 consiste en la suma en el sistema binario de los números 11001 y 11010. Esta suma es llevada a cabo mediante las dos tablas de la Figura 1: la tabla de la izquierda muestra el cálculo del acarreo, a lo que comúnmente nos referimos como "lo que se lleva" y en la tabla de la derecha se muestra el cálculo del dígito de la suma. En la primera columna de derecha a izquierda se calcula $1+0$ dando como resultado 1 y acarreo 0 (que no se apunta), en la segunda columna se calcula $0+1$ dando 1 y acarreo 0 , en la tercera columna se calcula $0+0$ obteniendo 0 con acarreo 0 ; en la cuarta columna $1+1$ da como resultado $10_{2}$, i.e., dígito 0 y acarreo 1 . Finalmente, $1+1+1=11_{2}$ de donde se sigue que la suma es 1 con acarreo igual a 1, ese acarreo solamente se escribió en la línea de resultados pues ya no había más elementos para sumar.

\section{Algoritmo para la suma en el sistema binario de números de tres dígitos}

El diseño de un dispositivo que realice la suma de dos números de varios dígitos en el sistema binario, precisa de una descripción del algoritmo de la suma donde todas las posibilidades estén contempladas. El método resultante es un poco más elaborado que el ejemplo ya descrito.

En seguida se muestra el procedimiento paso a paso, para sumar dos números binarios de tres dígitos cada uno, en el entendido de que es suficiente para generalizar a más dígitos. Aquí se hace un uso estricto de las tablas para la suma y para el acarreo mostradas en el ejemplo anterior.

Iniciamos con la suma de los primeros dígitos $d_{1}$ y $c_{1}$, dando como resultado $f_{1}$ para el dígito de la suma y, acarreo igual a $a_{1}$; la siguiente figura muestra el registro de estos resultados:

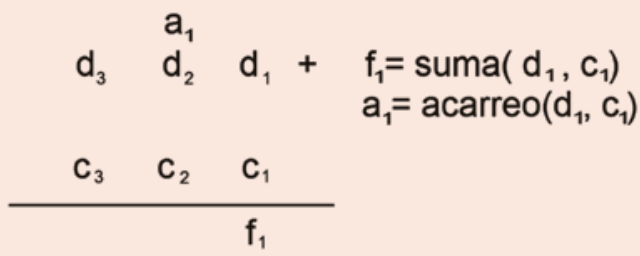

Figura 2. Suma de los primeros dígitos.
Luego, se calcula la suma parcial del acarreo $a_{1}$ con el dígito $d_{2}$ y se obtiene el dígito $s_{1}$ para la suma y un acarreo igual a $a_{2}$ :

$$
\begin{array}{llll}
\mathrm{d}_{3} & \begin{array}{c}
\mathrm{a}_{1} \\
\mathrm{~d}_{2} \\
\mathrm{a}_{2} \mathrm{~s}_{1}
\end{array} & \mathrm{~d}_{1}+\begin{array}{l}
\mathrm{s}_{1}=\operatorname{suma}\left(\mathrm{a}_{1}, \mathrm{~d}_{2}\right) \\
\mathrm{a}_{2}=\operatorname{acarreo}\left(\mathrm{a}_{1}, \mathrm{~d}_{2}\right)
\end{array} \\
\mathrm{c}_{3} & \mathrm{c}_{2} \quad \mathrm{c}_{1}
\end{array}
$$

Figura 3. Suma parcial y acarreo.

Sumando $s_{1}$ con el dígito $c_{2}$ obtenemos $f_{2}$ como dígito de la suma y acarreo $a_{3}$, como se muestra en la figura:

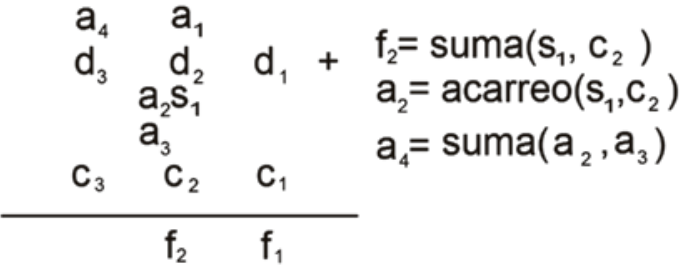

Figura 4. Suma de los segundos dígitos y acarreo.

Los acarreos recientes se suman entre sí para obtener el acarreo total $a_{4}=a_{2}+a_{3}$, es importante aclarar que esta suma de acarreos nunca genera un nuevo acarreo y no es difícil convencerse que $a_{2}$ y $a_{3}$ no toman el valor 1 simultáneamente.

El mismo procedimiento se repite sobre la siguiente columna, como se ilustra en la figura:

$$
\begin{aligned}
& a_{4} \quad a_{1} \quad s_{2}=\operatorname{suma}\left(a_{4}, d_{3}\right) \\
& d_{3} d_{2} d_{1}+a_{5}=\operatorname{acarreo}\left(a_{4}, d_{3}\right) \\
& \begin{array}{lll}
a_{5} s_{2} & a_{2} s_{1} & f_{3}=\operatorname{suma}\left(s_{2}, c_{3}\right) \\
a_{6} & a_{3} &
\end{array} \\
& \mathrm{C}_{3} \quad \mathrm{C}_{2} \quad \mathrm{C}_{1} \quad \mathrm{a}_{6}=\operatorname{acarreo}\left(\mathrm{s}_{2}, \mathrm{C}_{3}\right) \\
& \begin{array}{lllll}
\mathrm{f}_{4} & \mathrm{f}_{3} & \mathrm{f}_{2} & \mathrm{f}_{1}
\end{array} \quad \mathrm{f}_{4}=\operatorname{suma}\left(\mathrm{a}_{5}, \mathrm{a}_{6}\right)
\end{aligned}
$$

Figura 5. Suma con acarreo en el sistema binario.

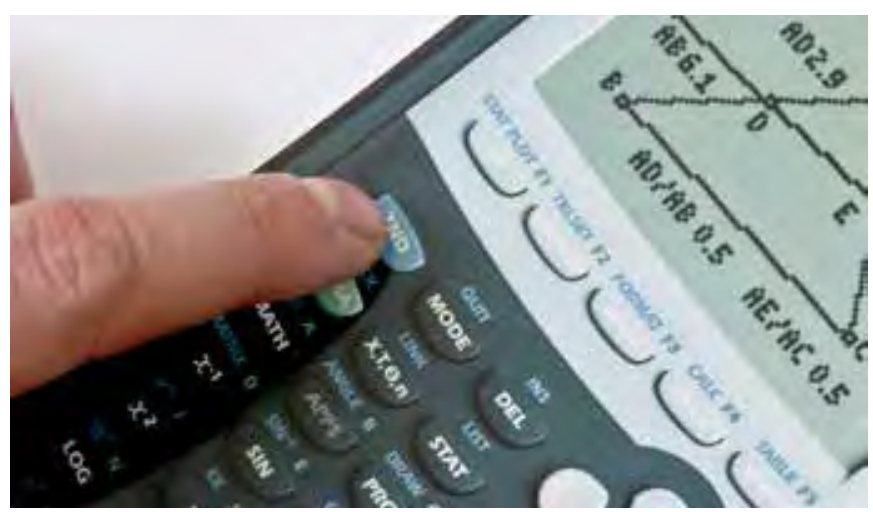


Retomando el ejemplo anterior (Figura 1), podemos incorporar en la operación las sumas parciales y acarreos previos. La siguiente figura muestra el registro de cada uno de los pasos descritos anteriormente.

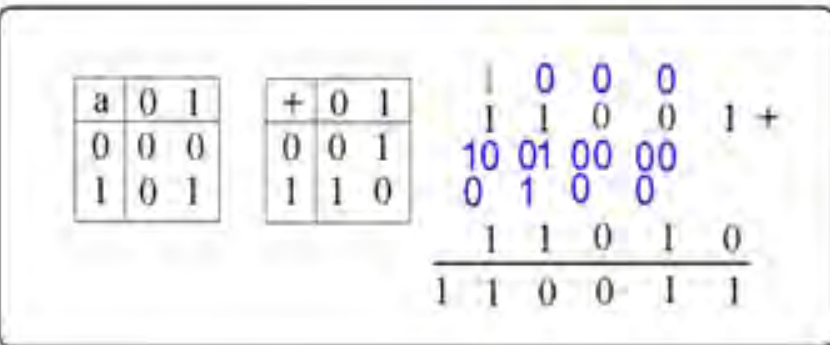

Figura 6. Ejemplo detallado de la suma de dos números binarios.

\section{ELEMENTOS PARA EL DISEÑO}

Es deseable comprender al menos un poco de circuitos lógicos, en particular sobre la conexión en serie y la conexión en paralelo, para darse cuenta que conectar de manera apropiada un par de interruptores para lograr el resultado deseado es una tarea simple.

Un interruptor de los que usamos en casa para encender una lámpara es un dispositivo mecánico muy sencillo. Consta de dos terminales donde conectan cables y una parte móvil que al accionarse pone en contacto físico dichas terminales y permite que la energía eléctrica fluya a través de él. El esquema para un interruptor como el mencionado se muestra en la siguiente figura:

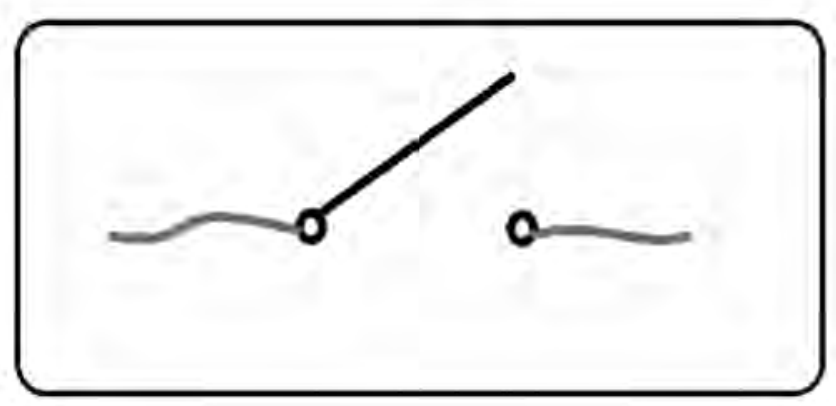

Figura 7. Representación de un interruptor.

Ésta es la versión más sencilla de un interruptor y se le conoce como interruptor de un polo, un tiro, dos posiciones: abierto y cerrado. Sin embargo para nuestros fines son necesarios interruptores no tan simples, como los que describimos a continuación.

En hogares de más de una planta suele haber una lámpara que ilumina las escaleras, donde el usuario puede encender o apagar la lámpara desde el pie de la escalera o desde la parte alta. Para construir el circuito correspondiente se requiere de interruptores de un polo, dos tiros y dos posiciones, el esquema se observa en la Figura 8.

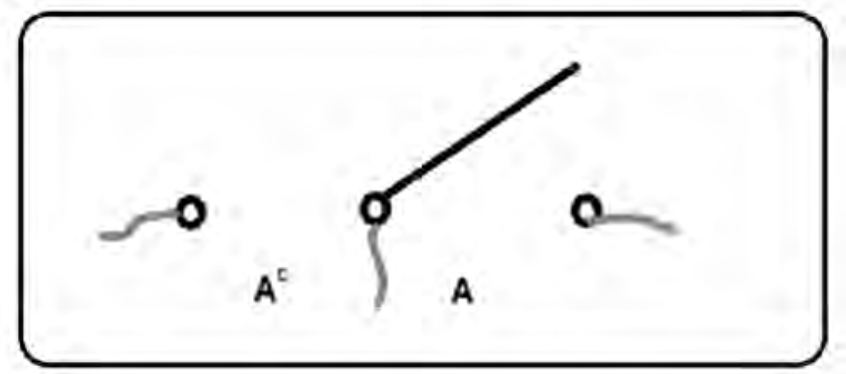

Figura 8. Interruptor un polo dos tiros dos posiciones.

En realidad se trata de dos interruptores simples acoplados, y se identifican con A y $\mathrm{A}^{\mathrm{C}}$ pues si la corriente entra por el nodo central saldrá ya sea por $A$ o por $\mathrm{A}^{\mathrm{C}}$ pues es de dos posiciones; así, si $A$ esta cerrado $(A=1), \mathrm{A}^{\mathrm{C}}$ estará abierto $(A=0)$ y viceversa. Existen dos formas de conectar dos interruptores de este tipo para controlar la lámpara de la escalera ya mencionada, una de estas formas está dada por el diagrama de la Figura 9.

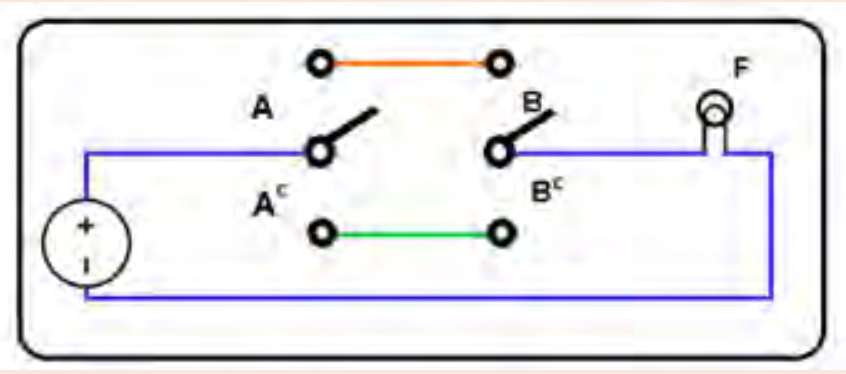

Figura 9. Circuito de la escalera I.

En este diagrama se observa que para que la lámpara encienda, $A$ y $B$ deben estar ambos cerrados $(A=1$ y $B=1)$ o ambos abiertos ( $A=0$ y $B=0$ ). Evidentemente, si sucede que $(A=1$ y $B=0)$ o que ( $A=0$ y $B=1)$ la lámpara estará apagada.

Las lámparas utilizadas para nuestro prototipo son diodos emisores de luz conocidos como LEDS.

Es necesario mostrar dos tipos de conexiones que dan lugar a expresiones para representar un circuito.

\section{Conexiones en serie y en paralelo}

Dos interruptores se dice están conectados en serie, cuando la salida de un interruptor está conectada a la entrada del otro interruptor (Figura 10).

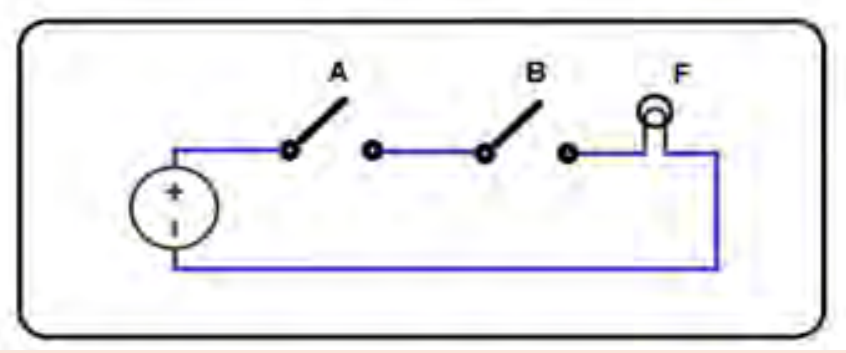

Figura 10. Conexión en serie. 
Se caracteriza porque es necesario que ambos interruptores estén cerrados $(A=1$ y $B=1)$ para que la lámpara esté encendida $(F=1)$. Simbólicamente, esto se representa como $F=A \wedge B$, al igual que se hace con la intersección de conjuntos. Para una exposición más completa de la teoría y aritmética de conjuntos, se puede consultar [7] y [8].

Dos interruptores se dicen estar conectados en paralelo si sus entradas están conectadas entre sí y también sus salidas están conectadas entre sí (Figura 11).

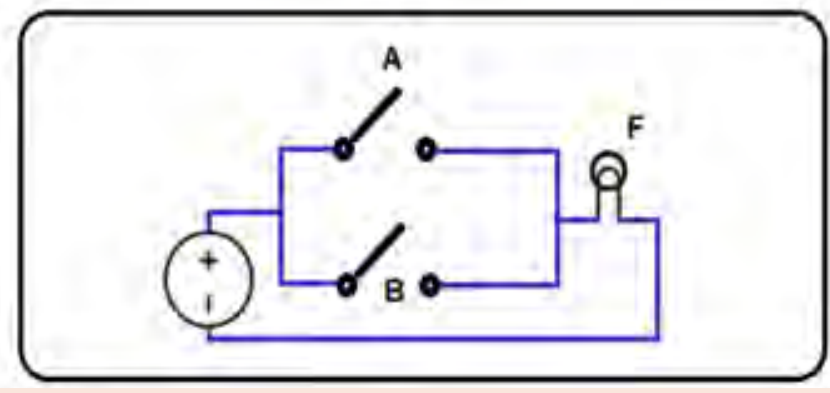

Figura 11. Conexión en paralelo.

Para que la lámpara $F$ esté encendida basta que uno de los interruptores este cerrado $(A=1 \circ B=1)$. Esta conexión se representa en símbolos por $F=A \vee B$ pues tiene el mismo comportamiento que la unión de conjuntos.

Es posible combinar los dos tipos de conexiones anteriores para generar comportamientos con mayor variedad, así el diagrama de escalera de la Figura 8 es una conexión mixta donde se observa que $A$ y $B$ están conectados en serie, $A^{c}$ y $B^{c}$ también están conectados en serie, a su vez la conexión $A \wedge B$ y la conexión $A^{c} \wedge B^{c}$ están conectadas en paralelo, en otras palabras la expresión para el circuito es $F=(A \wedge B) \vee\left(A^{c} \wedge B^{c}\right)$.

A la conexión $A \wedge B$ se le conoce como compuerta AND y a la expresión $A \vee B$ se le conoce como la compuerta OR.

Existe otra forma de construir el circuito de la escalera y se caracteriza por funcionar totalmente opuesto al circuito de la escalera I (Figura 8), esto es, ahora la lámpara estará encendida si $A$ y $B$ toman valores contrarios y estará apagada si $A$ y $B$ toman valores iguales. El diagrama correspondiente se muestra en la Figura 12.

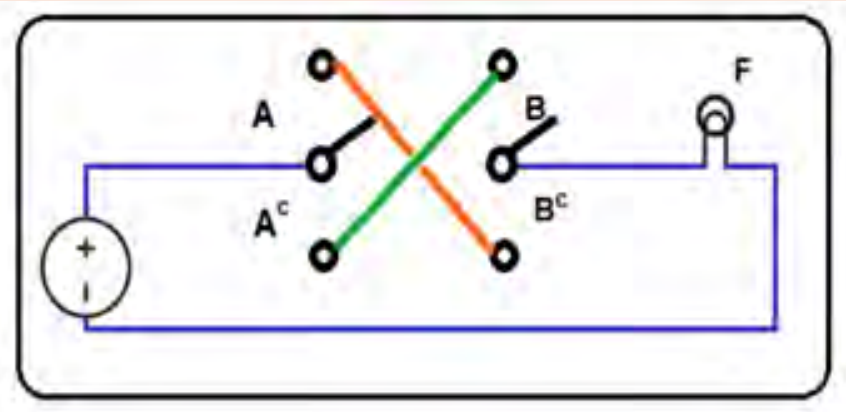

Figura 12. Circuito de la escalera II.
La expresión para este circuito es $F=\left(A \wedge B^{c}\right) \vee\left(A^{c} \wedge B\right)$ y recibe el nombre de compuerta XOR.

\section{SUMADORA}

En este circuito se utilizan interruptores de dos polos dos tiros y dos posiciones.

En la Figura 13 se muestra el circuito que realiza la suma de dos dígitos binarios. Es necesario precisar que las lámparas $c_{1}$ y $d_{1}$ nos indicarán si los interruptores se activarán o no.

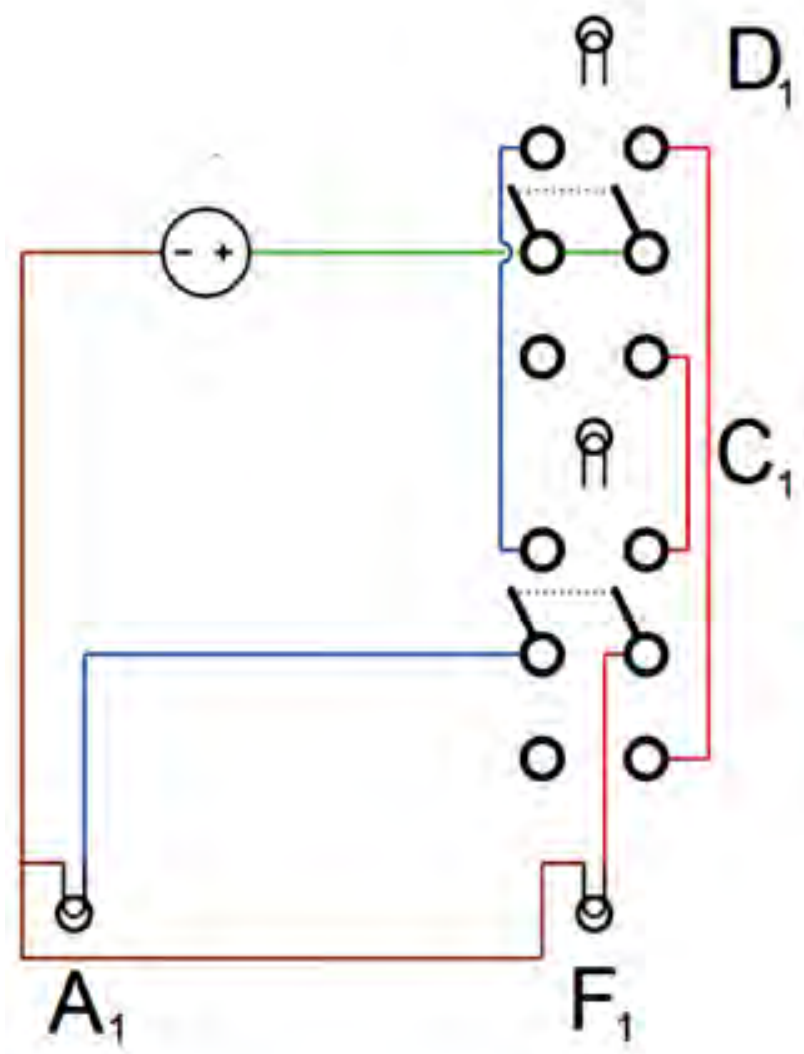

Figura 13. Circuito semisumador.

Podemos afirmar que cada par Lámpara-Interruptor constituye un relevador manual, esto es: la señal luminosa nos indica que el interruptor deberá ser deslizado hacia ella. Es deseable evitar el uso de relevadores automáticos que pueden restar credibilidad al instrumento y aumentar su costo. Hasta esta parte el relevador manual tiene un uso poco significativo, sin embargo en el diseño del prototipo completo juega un papel medular.

Nótese que para calcular el dígito de la suma de $c_{1}$ con $d_{1}$ se utiliza una compuerta XOR representada por $f_{1}=\left(d_{1} \wedge\right.$ $\left.c_{1}^{c}\right) \vee\left(d_{1}^{c} \wedge c_{1}\right)$, de hecho es idéntica a la segunda versión mostrada del circuito de la escalera (Figura 12). También se observa que para obtener el acarreo simplemente hay que conectar en serie a $c_{1}$ con $d_{1}$, esto es: $a_{1}=c_{1} \wedge d_{1}$.

Una vez entendido el circuito semisumador, mencionamos los aspectos necesarios para comprender 
el funcionamiento del sumador de números de dos o más dígitos binarios. El diagrama se muestra en la Figura 14. Es importante aclarar que la distribución de los interruptores ha sido levemente modificada a fin de que los relevadores manuales correspondientes a sumas parciales y algunos de los acarreos queden todos en la parte superior y con ello facilitar la operación.

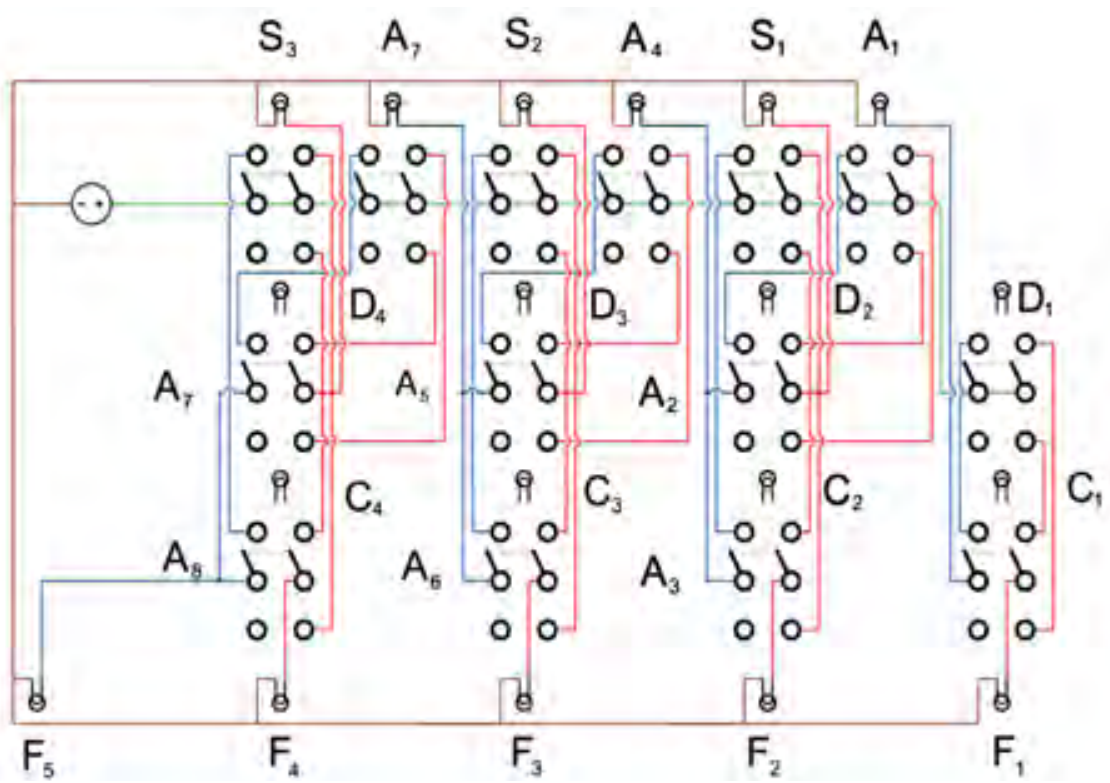

Figura 14. Circuito para sumar dos números de cuatro dígitos binarios.

Los pares de lámpara e interruptor que aparecen en la parte superior del instrumento son relevadores manuales muy importantes en el diseño propuesto, de modo que deben ser accionados (desplazados) por el usuario de acuerdo al estado de la lámpara correspondiente: encendida o apagada. Una lámpara encendida nos indica un resultado parcial, ya sea de un acarreo o del dígito de la suma. De esta manera, una vez introducidos los dos sumandos se accionan los interruptores según indiquen las lámparas $D_{i}$ y $C_{i}$, revisamos las lámparas superiores de derecha a izquierda y si una lámpara está encendida, entonces accionamos el interruptor correspondiente. Puede ser que las lámparas de la izquierda cambien su estado al accionar algún interruptor de la derecha.

Un detalle importante es que los acarreos que resultan de sumar dos acarreos son muy simples de obtener. Se puede probar sin mucha dificultad, como ya lo habíamos mencionado, que los acarreos a sumar no pueden ser ambos iguales a 1 al mismo tiempo, de esta manera simplemente hay que unir los cables para enviar la señal eléctrica pues basta que uno tenga corriente eléctrica para que la suma sea uno. Nótese que $F_{5}$ se calcula de esa manera.

Con el fin de que el usuario no requiera de la consulta de las equivalencias entre el sistema decimal y el sistema binario, introducimos dos circuitos especiales: un codificador, que convierte un número dado en el sistema decimal a su expresión en el sistema binario y, un decodificador, que convierte el resultado obtenido por la suma en el sistema binario al sistema decimal.

\section{Circuito codificador}

Construir el circuito que traduzca números dados en sistema decimal a sistema binario no es una tarea sencilla. En condiciones normales la traducción se realiza utilizando el algoritmo de expansión que consiste de realizar multiplicaciones y sumas de manera apropiada, un procedimiento más

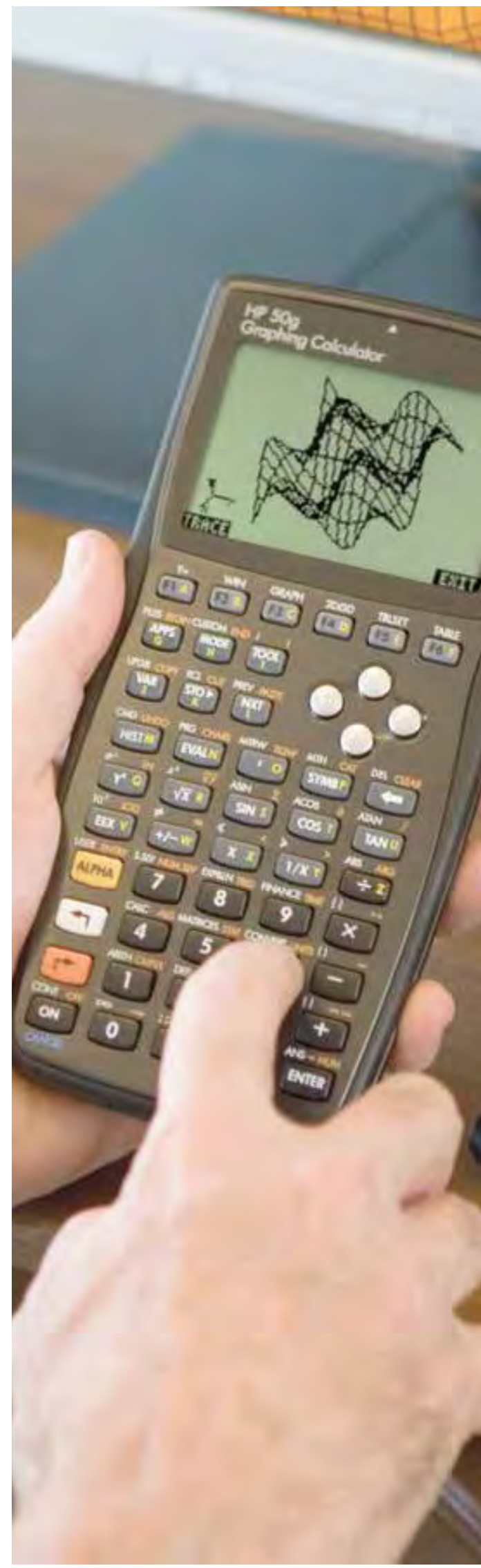


elaborado que el procedimiento de suma que se pretende exhibir. Además el circuito lógico correspondiente es más complicado que el circuito lógico para sumar números binarios.

Afortunadamente se ha encontrado la forma de construir un circuito lógico mucho más sencillo que el anterior. Ayuda enormemente acotar los números decimales a introducir; del cero al diez, si el circuito se desea realizar en base a interruptores solo se requieren interruptores de tres polos, pues los números binarios correspondientes a los decimales ocupan a lo más tres dígitos binarios. Es difícil encontrar interruptores de tres polos en el mercado, pero gracias a la popularización de las herramientas de corte con control numérico (para una mayor información sobre los sistemas de control numérico puede consultar [9]) y a la disponibilidad de programas CAD para el diseño, se pueden fabricar con relativa facilidad incluso de muchos más polos. El circuito estará elaborado con interruptores de cinco polos, un polo será utilizado para hacer evidente el número decimal que se está introduciendo. $Y$ aunque sobren polos, es preferible que los mismos polos no utilizados reflejen que no se ocupan, además fabricándolos de dos tiros es posible reducir el número de interruptores y el espacio. Es importante resaltar que en este circuito codificador los interruptores serán de cinco polos dos tiros y tres posiciones, en los otros circuitos todos serán de dos posiciones, dos tiros y varían la cantidad de polos.

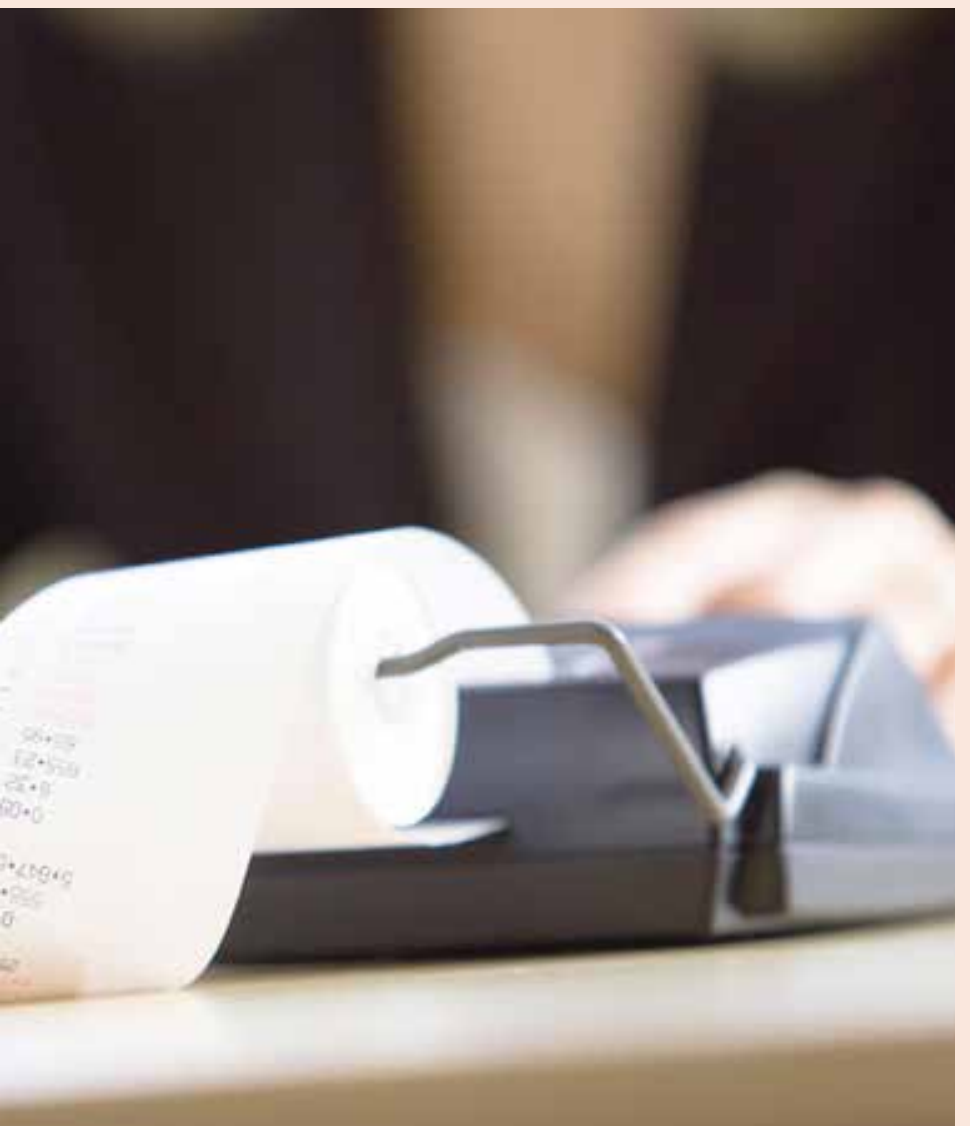

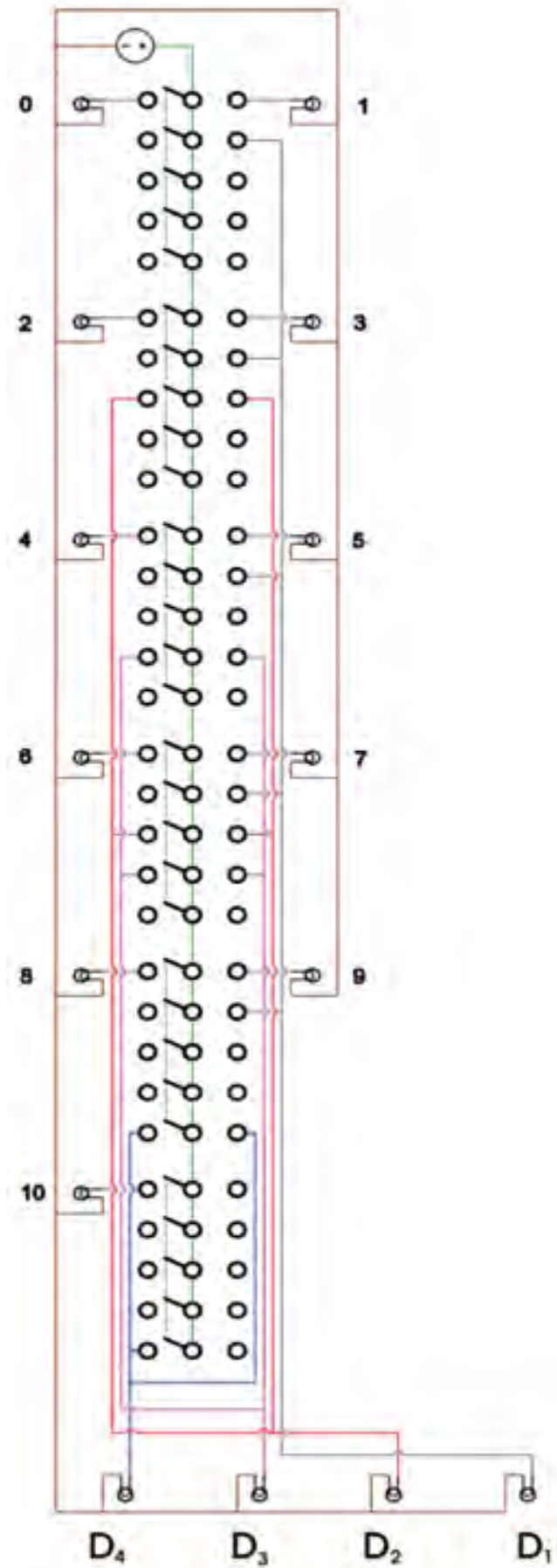

Figura 15. Circuito codificador

Observando la Tabla 1 de las equivalencia entre el sistema decimal y el sistema binario, podemos deducir qué lámparas deben encenderse cuando se acciona de manera única el interruptor correspondiente a alguno de los números a sumar; por ejemplo, si se acciona el interruptor correspondiente al dígito 5 , se encenderá la lámpara precisamente a un lado del $5 y$, dado que la secuencia en el sistema binario para el 5 es 0101, se deben encender sólo 
las lámparas $D_{3}$ y $D_{1}$. Así, sólo accionando los interruptores correspondientes a los números impares pueden encender la lámpara $D_{1}$; los interruptores correspondientes a $2,3,6$, 7 y 10 pueden encender la lámpara $D_{2}$ y 4, 5, 6 y 7 pueden encender la lámpara $D_{3}$, los interruptores de los números 8,9 y 10 mandan a encender la lámpara $D_{4}$. Es necesario especificar que en el circuito para funcionar de manera adecuada sólo hay que activar un interruptor a la vez, para introducir el segundo sumando se requiere de otro circuito idéntico. De esta manera al activar un interruptor se enviará al circuito sumador una secuencia de cuatro dígitos binarios $D_{4}, D_{3}, D_{2}$ y $D_{1}$ para el primer sumado y con el otro circuito se enviará la secuencia $c_{1}, c_{2}, c_{3}$ y $c_{4}$ visualizándose como lámparas encendidas o pagadas. El usuario debe accionar hacia arriba a los interruptores que tengan una lámpara encendida.

\section{Circuito decodificador}

Una vez obtenida la suma en el sistema binario dada por la secuencia $\left(F_{5}, F_{4}, F_{3}, F_{2}, F_{1}\right)$ es deseable visualizar el resultado en nuestro conocido sistema decimal, el método normal para llevar a cabo esta traducción utiliza en cada uno de sus pasos al algoritmo de la división, procedimiento más complicado que el procedimiento que deseamos resaltar, para saber más se puede consultar Karris [10] y Holdsworth y Woods [11]. Afortunadamente, debido a las restricciones introducidas y a la posibilidad de fabricar interruptores con la cantidad necesaria de polos, es posible diseñar un circuito que realice la tarea sin recurrir a algoritmos complicados. Nos limitaremos señalar que el circuito tiene forma de árbol, en la Figura 16 se puede constatar que las secuencias $(0,0,0,0,0),(0,0,0,0,1),(0,0,0,1,0)$ y $(0,0,0,1,1)$ encienden las lámparas correspondientes a las sumas 0,1 , 2 y 3.

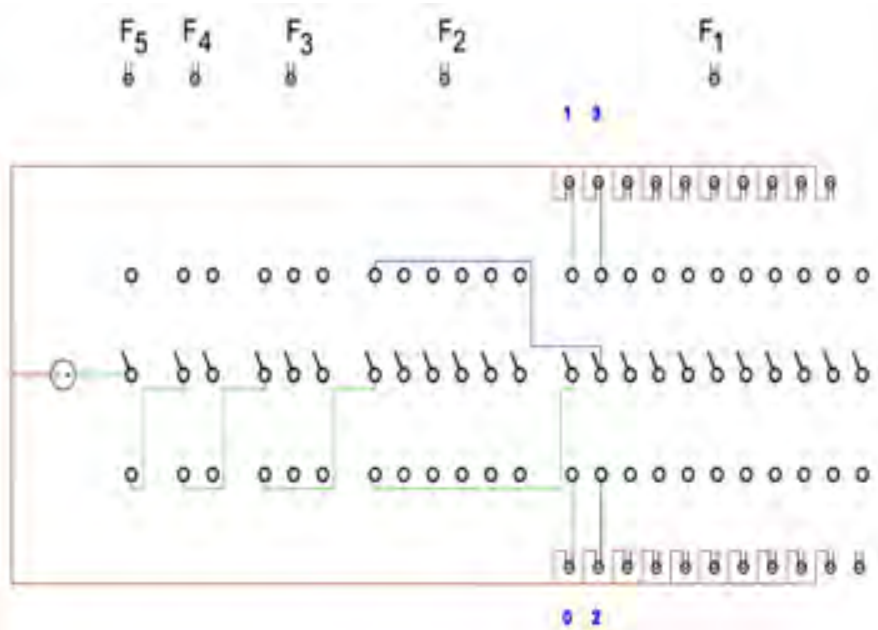

Figura 16. Decodificador para 0, 1, 2 y 3.

De la misma manera sucede para las secuencias $(0,0,1,0,0),(0,0,1,0,1),(0,0,1,1,0)$ y $(0,0,1,1,1)$ para las sumas $4,5,6$ y 7 .

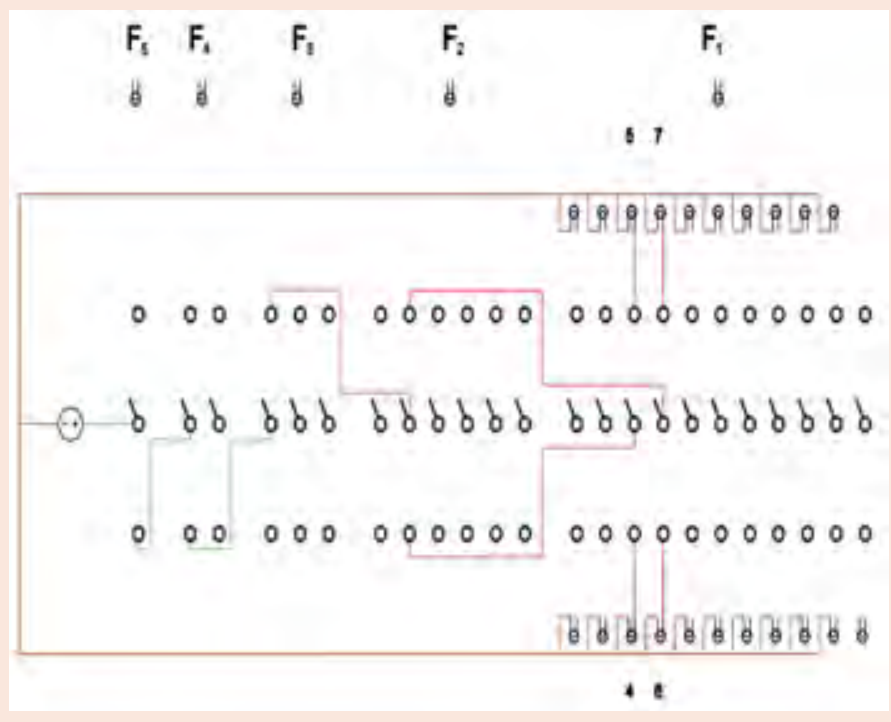

Figura 17. Decodificador para 4, 5, 6 y 7.

Para mostrar que el circuito funciona, verifique que cuando sólo uno de los interruptores correspondientes a los dígitos 0, 1, 2 o 3 es activado, se enciende la lámpara correspondiente.

El siguiente circuito tiene la capacidad de convertir una secuencia de cinco dígitos binarios al número decimal correspondiente. Observe que fue necesario utilizar interruptores con una cantidad de polos poco común; en caso de haber diseñado nuestro instrumento para recibir números más grandes que diez, eso implicaría la necesidad de incrementar aún más la cantidad de polos.

Para operar el circuito es necesario activar los interruptores según lo indique la secuencia $F_{5}, F_{4}, F_{3}, F_{2}$, $F_{1}$, esto es si alguna lámpara está encendida el interruptor deberá deslizarse hacia ella y, si está apagada deberá alejarse de él.

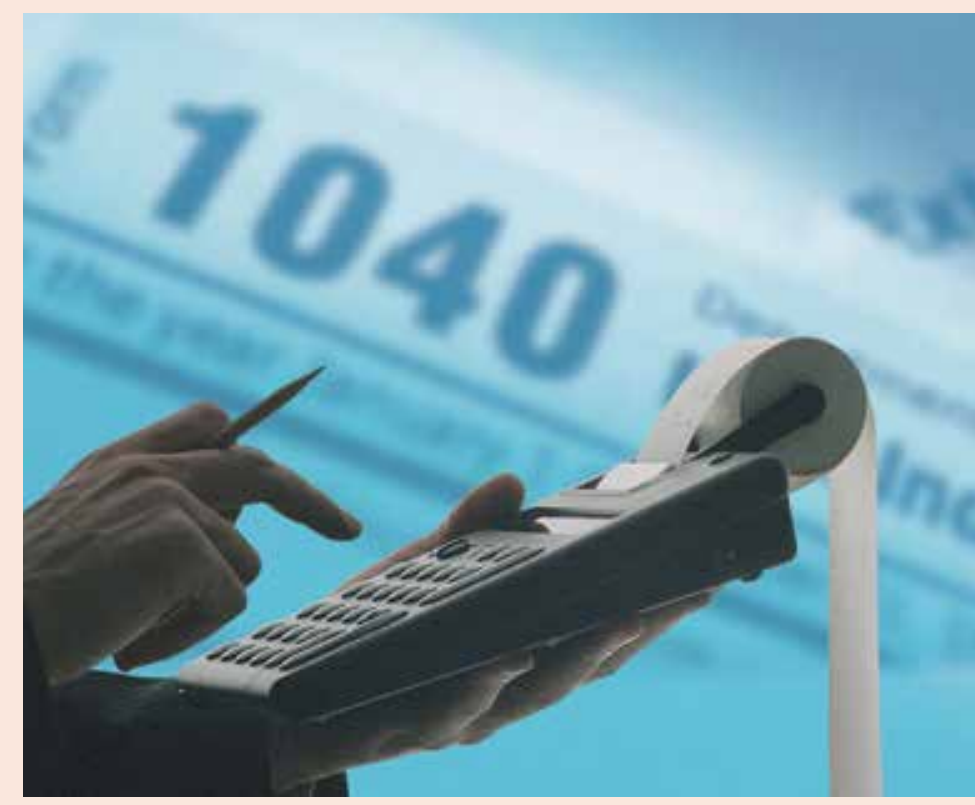




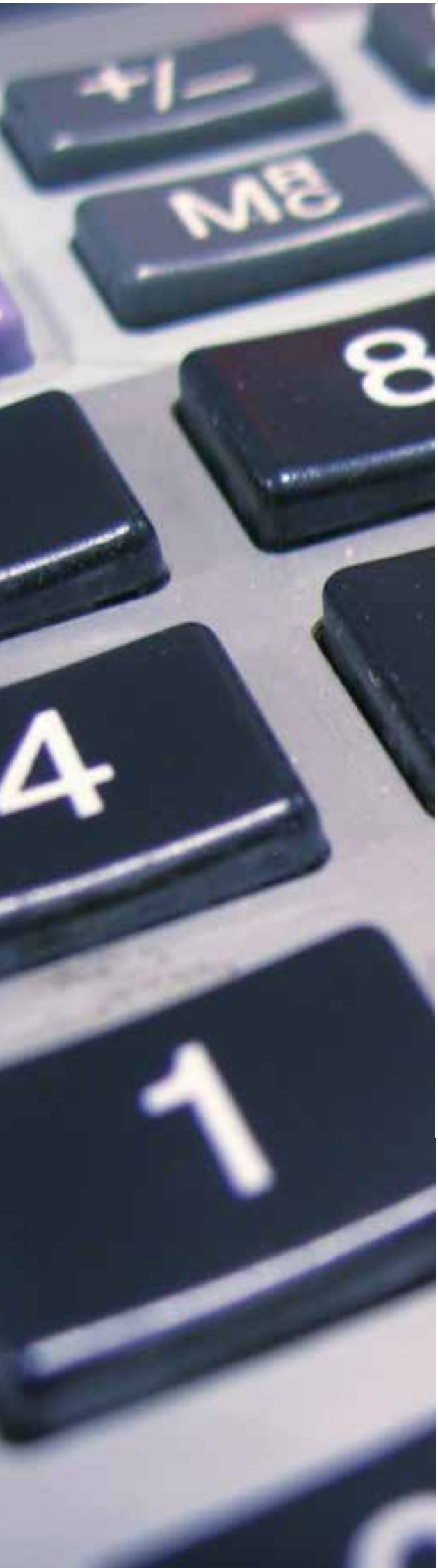

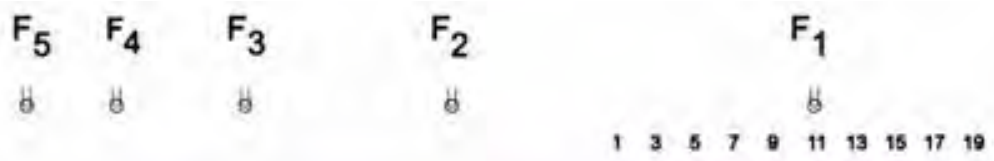

ดคดคดคดคดค

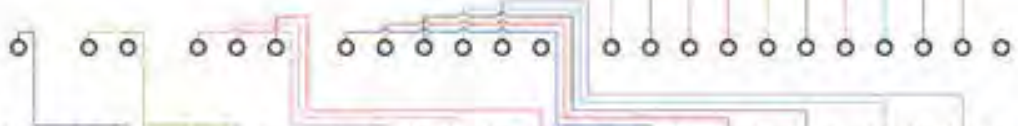

b bb bbb bbbbbb bbbbbbbbbbb

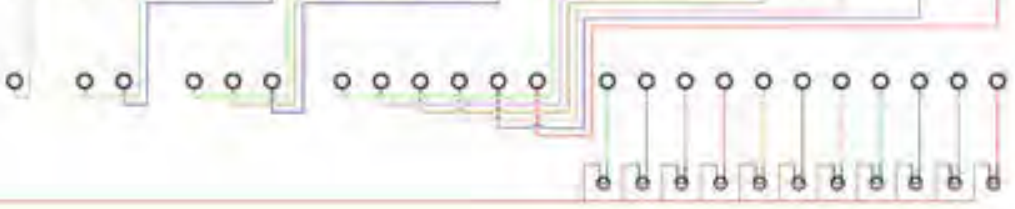

Figura 18. Circuito decodificador.

Se puede verificar el funcionamiento utilizando la tabla de la Figura 1. Las expresiones para cada uno de los resultados están dadas en la siguiente tabla.

Tabla 2. Expresiones de las funciones del decodificador.

\begin{tabular}{|l|l|}
\hline$R_{0}=\left(F_{5}^{c}\right) \wedge\left(F_{4}^{c}\right) \wedge\left(F_{3}^{c}\right) \wedge\left(F_{2}^{c}\right) \wedge\left(F_{1}^{c}\right)$ & $R_{10}=\left(F_{5}^{c}\right) \wedge\left(F_{4}\right) \wedge\left(F_{3}^{c}\right) \wedge\left(F_{2}\right) \wedge\left(F_{1}^{c}\right)$ \\
\hline$R_{1}=\left(F_{5}^{c}\right) \wedge\left(F_{4}^{c}\right) \wedge\left(F_{3}^{c}\right) \wedge\left(F_{2}^{c}\right) \wedge\left(F_{1}\right)$ & $R_{11}=\left(F_{5}^{c}\right) \wedge\left(F_{4}\right) \wedge\left(F_{3}^{c}\right) \wedge\left(F_{2}\right) \wedge\left(F_{1}\right)$ \\
\hline$R_{2}=\left(F_{5}^{c}\right) \wedge\left(F_{4}^{c}\right) \wedge\left(F_{3}^{c}\right) \wedge\left(F_{2}\right) \wedge\left(F_{1}^{c}\right)$ & $R_{12}=\left(F_{5}^{c}\right) \wedge\left(F_{4}\right) \wedge\left(F_{3}\right) \wedge\left(F_{2}^{c}\right) \wedge\left(F_{1}^{c}\right)$ \\
\hline$R_{3}=\left(F_{5}^{c}\right) \wedge\left(F_{4}^{c}\right) \wedge\left(F_{3}^{c}\right) \wedge\left(F_{2}\right) \wedge\left(F_{1}\right)$ & $R_{13}=\left(F_{5}^{c}\right) \wedge\left(F_{4}\right) \wedge\left(F_{3}\right) \wedge\left(F_{2}^{c}\right) \wedge\left(F_{1}\right)$ \\
\hline$R_{4}=\left(F_{5}^{c}\right) \wedge\left(F_{4}^{c}\right) \wedge\left(F_{3}\right) \wedge\left(F_{2}^{c}\right) \wedge\left(F_{1}^{c}\right)$ & $R_{14}=\left(F_{5}^{c}\right) \wedge\left(F_{4}\right) \wedge\left(F_{3}\right) \wedge\left(F_{2}\right) \wedge\left(F_{1}^{c}\right)$ \\
\hline$R_{5}=\left(F_{5}^{c}\right) \wedge\left(F_{4}^{c}\right) \wedge\left(F_{3}\right) \wedge\left(F_{2}^{c}\right) \wedge\left(F_{1}\right)$ & $R_{15}=\left(F_{5}^{c}\right) \wedge\left(F_{4}\right) \wedge\left(F_{3}\right) \wedge\left(F_{2}\right) \wedge\left(F_{1}\right)$ \\
\hline$R_{6}=\left(F_{5}^{c}\right) \wedge\left(F_{4}^{c}\right) \wedge\left(F_{3}\right) \wedge\left(F_{2}\right) \wedge\left(F_{1}^{c}\right)$ & $R_{16}=\left(F_{5}\right) \wedge\left(F_{4}^{c}\right) \wedge\left(F_{3}^{c}\right) \wedge\left(F_{2}^{c}\right) \wedge\left(F_{1}^{c}\right)$ \\
\hline$R_{7}=\left(F_{5}^{c}\right) \wedge\left(F_{4}^{c}\right) \wedge\left(F_{3}\right) \wedge\left(F_{2}\right) \wedge\left(F_{1}\right)$ & $R_{17}=\left(F_{5}\right) \wedge\left(F_{4}^{c}\right) \wedge\left(F_{3}^{c}\right) \wedge\left(F_{2}^{c}\right) \wedge\left(F_{1}\right)$ \\
\hline$R_{8}=\left(F_{5}^{c}\right) \wedge\left(F_{4}\right) \wedge\left(F_{3}^{c}\right) \wedge\left(F_{2}^{c}\right) \wedge\left(F_{1}^{c}\right)$ & $R_{18}=\left(F_{5}\right) \wedge\left(F_{4}^{c}\right) \wedge\left(F_{3}^{c}\right) \wedge\left(F_{2}\right) \wedge\left(F_{1}^{c}\right)$ \\
\hline$R_{9}=\left(F_{5}^{c}\right) \wedge\left(F_{4}\right) \wedge\left(F_{3}^{c}\right) \wedge\left(F_{2}^{c}\right) \wedge\left(F_{1}\right)$ & $R_{19}=\left(F_{5}\right) \wedge\left(F_{4}^{c}\right) \wedge\left(F_{3}^{c}\right) \wedge\left(F_{2}\right) \wedge\left(F_{1}\right)$ \\
\hline
\end{tabular}

Puede verificar que la suma 20, se obtiene con la expresión $R_{20}=\left(F_{5}\right) \wedge\left(F_{4}^{c}\right) \wedge$ $\left(F_{3}\right) \wedge\left(F_{2}^{c}\right) \wedge\left(F_{1}^{c}\right)$, este resultado se obtiene cuando $F_{5}=1, F_{4}=0, F_{3}=1, F_{2}=0$ y $F_{1}$ $=0$, pues la secuencia correspondiente al número 20 en sistema binario es 10100 . Partes del prototipo

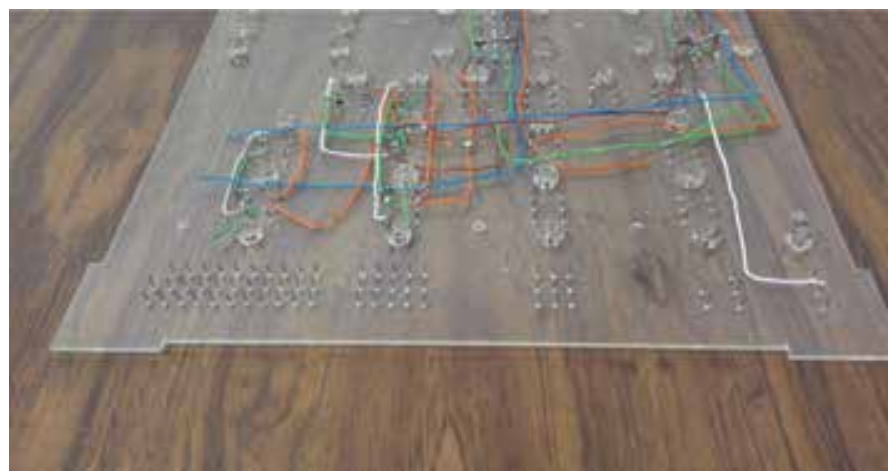

Figura 19. Vista trasera de las terminales del codificador.

Las terminales son fragmentos de alfileres con cabeza que se insertan en orificios previamente realizados con el radio necesario para que los alfileres 
entren a presión y se detengan en las terminales, en donde se conectan los cables que dan forma a cada circuito.

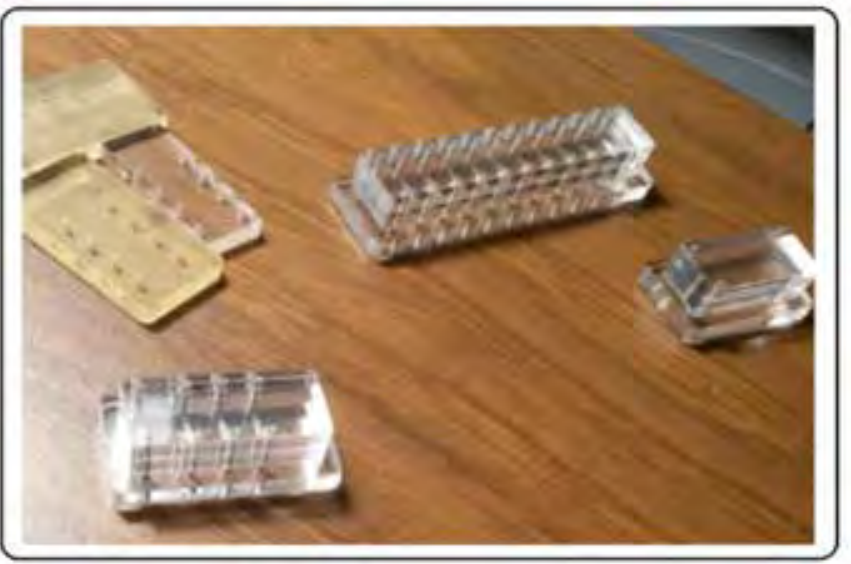

Figura 20. Elementos deslizantes.

Los elementos deslizantes están formados por tres capas de rectángulos con orificios para insertar grapas de tal manera que estas produzcan el contacto físico entre las terminales cuando los elementos deslizantes son arrastrados hasta uno de sus extremos

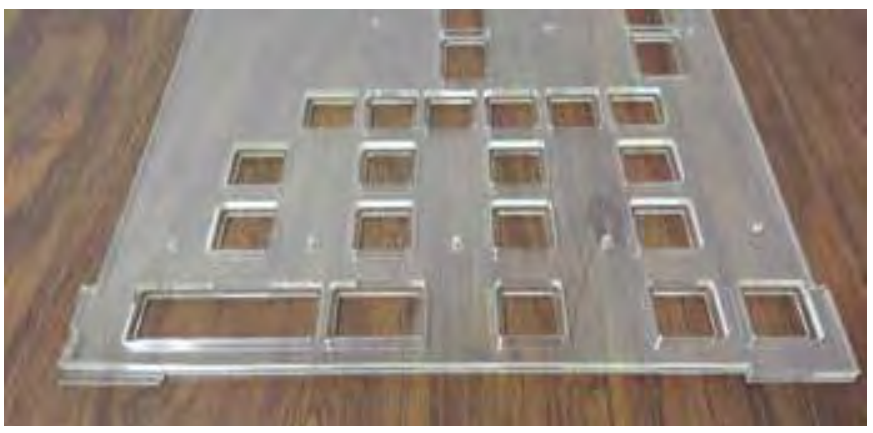

Figura 21. Placa cajón para elementos deslizantes.

La placa cajón permite que el elemento deslizante tenga la libertad de movimiento en un solo sentido sin que se pierda el contacto con las terminales que yacen en la parte de abajo.

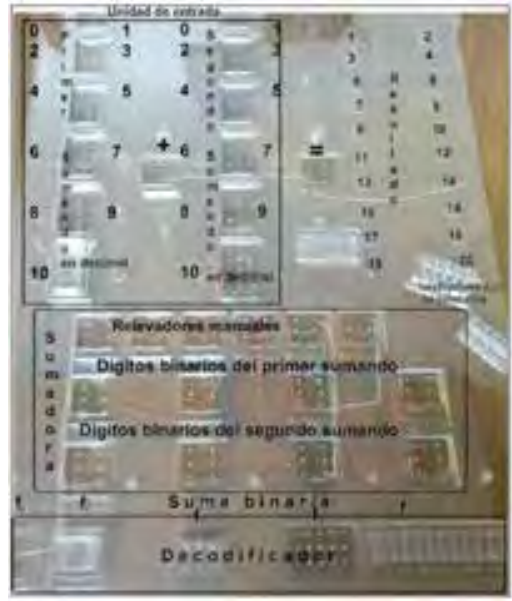

Figura 22. Estado del prototipo.

\section{Resumen de la operación:}

- El usuario enciende el dispositivo donde se encuentra un signo" +".

- Luego activa dos interruptores del área de entrada, según sea la elección de los sumandos.

- Se fija en el estado de las lámparas donde se indican los dígitos en binario de los sumandos y activan los interruptores correspondientes, en ese momento ha introducido los sumandos a la sumadora.

- Revisa el resultado obtenido de cinco dígitos binarios y activa los interruptores correspondientes de la unidad decodificadora.

- Por último activa el interruptor que esta junto al símbolo " $=$ ", y se debe observar que enciende la lámpara correspondiente al resultado correcto.

\section{CONCLUSIONES}

Se ha logrado construir un prototipo didáctico que permite una alta interacción con el usuario en el proceso de cómputo, que en este caso corresponde a la suma. Además, le permite al usuario visualizar claramente el proceso interno, lo cual propicia un mayor entendimiento del cómputo aritmético a través de circuitos eléctricos y, la utilización de elementos sencillos aumenta la confianza de que no existen trampas ni aspectos obscuros presentes en el prototipo.

El hecho de haber evitado el uso de relevadores automáticos obliga a que el usuario sea partícipe del procedimiento de cálculo, de manera muy similar a cuando se llega a realizar una tarea en una computadora donde el usuario toma decisiones importantes para lograr su objetivo.

\section{BIBLIOGRAFÍA}

[1]T. Infante y M. Benítez, “Historia de la computación y estructura de un computador". En línea, recuperado el 3 de noviembre de 2016 en: http://www.ilustrados.com/tema/894/Historiacomputacion-estructura-computador.html

[2] Peter Norton, "Introduction to Computers", Concepts Series, 9th edition, McGraw-Hill, 2008.

[3] N. Balabanian and B. Carlson, "Digital Logic Design Principles", Wiley India Pvt. Limited, 2007.

[4] M. Morris Mano and Michael D. Ciletti, "Digital Design with An Introduction to the Verilog HDL", Pearson Publishers, Fifth Edition, 2013.

[5] A. Glaser, "History of binary and other nondecimal numeration", Anton Glaser Publisher, 1971.

[6] J. E. Whitesitt, "Boolean Algebra and Its Applications", Reading, Mass: Addison-Wesley, 1961.

[7] T. Jech, "Set Theory: The Third Millennium Edition, revised and expanded". Springer Monographs in Mathematics, 2006.

[8] S. Lipschutz, "Schaum's Outline of Set Theory and Related Topics". Schaum's Series, McGraw-Hill Education, 1998.

[9] J. Francis Reintjes, "Numerical Control: Making a New Technology". Oxford Series on Advanced Manufacturing, Oxford University Press, 1991.

[10] Steven T. Karris, "Digital Circuit Analysis and Design with Simulink Modeling and Introduction to CPLDs and FPGAs". Orchard Publications, 2007.

[11] B. Holdsworth and C. Woods, "Digital Logic Design”. Newnes, 4th edition, revised. 2002. 